\title{
Effects of ethanol on threshold and duration of amygdaloid kindled seizures
}

\author{
ERNEST D. KEMBLE, THOMAS J. SKOGLUND, and VICKI A. DAVIES \\ University of Minnesota, Morris, Minnesota 56267.
}

\begin{abstract}
Susceptibility to amygdaloid kindled seizures in rats was examined after injections of isotonic saline or $.8 \mathrm{~g} / \mathrm{kg}$ or $1.6 \mathrm{~g} / \mathrm{kg}$ ethanol. Ethanol produced a dose-dependent increase in threshold for Stage 4-5 seizures and decreased seizure duration. Thresholds for Stage 1-3 seizures were also elevated by ethanol.
\end{abstract}

Ethanol produces a transient (approximately $3.5-\mathrm{h}$ ) decrease in the duration of both motor seizures and afterdischarges evoked by amygdaloid stimulation in previously kindled rats (Mucha \& Pinel, 1979). This is followed by potentiation of these stimulation effects. Further, Pinel, Van Oot, and Mucha (1975) have also shown that prior kindling intensifies alcohol withdrawal symptoms in rats. It therefore seemed of interest to further explore the interaction of ethanol and kindling phenomena. The present experiment examines the effect of two ethanol doses on the threshold and duration of amygdaloid kindled motor seizures.

\section{METHOD}

\section{Subjects}

The subjects were 15 female albino rats (Holtzman Company) weighing 319-382 $\mathrm{g}$ at the time of testing. All subjects had been previously kindled with 1 -sec trains of 200-microA biphasic pulses (60 pps) to a criterion of four consecutive Stage 4 or 5 seizures (Racine, 1972). This preliminary testing was completed $24 \mathrm{~h}$ prior to the present experiment. Subjects were individually housed and had ad-lib access to food (Purina Rat Chow) and water except during testing.

\section{Surgery and Histology}

All surgery was conducted under clean conditions employing Chloropent anesthesia $(2.0 \mathrm{ml} / \mathrm{kg})$ supplemented by Xylocaine and preoperative atropine. All subjects received postoperative penicillin $(100,000 \mathrm{U})$. Bipolar electrodes were constructed of two twisted strands of .2-1mm-diam stainless steel wire insulated with Epoxylite except at the cut ends. The electrodes were implanted (flat skull coordinates) $2.5 \mathrm{~mm}$ posterior to bregma, $4.0 \mathrm{~mm}$ left of the midline, and $7.4 \mathrm{~mm}$ ventral to the cortical surface.

At the conclusion of the testing, 40-micron coronal sections through the electrode site were prepared and stained with thionin for histological examination.

\section{Apparatus}

Testing was conducted in a $30.5 \times 28.5 \times 22.6 \mathrm{~cm}$ clear Plexiglas chamber. The floor consisted of $.6-\mathrm{cm}$-diam steel rods placed $1.25 \mathrm{~cm}$ apart. Biphasic stimulation was delivered by a Grass stimulator (Model S-4), and current intensity was monitored with a Tektronix (Model 502A) oscilloscope.

\section{Procedure}

Motor seizures elicited by stimulation were scored according to the five-stage classification suggested by Racine (1972). Each rat received three consecutive threshold tests at 24-h intervals Thresholds for Stage 4 or 5 motor seizures were determined $30 \mathrm{~min}$ after $8.0-\mathrm{ml} / \mathrm{kg}$ ip injections of isotonic saline, $10 \% \mathrm{v} / \mathrm{v}$ ethanol $/$ saline $(.8 \mathrm{~g}$ ethanol $/ \mathrm{kg})$, and $20 \% \mathrm{v} / \mathrm{v}$ ethanol $/$ saline $(1.6 \mathrm{~g}$ ethanol $/ \mathrm{kg})$. The order of drug administration was balanced among subjects. Thirty minutes after each drug injection, the rat was placed in the testing chamber and allowed to adapt for $2 \mathrm{~min}$. Stimulation trains $(1.0 \mathrm{sec}$ of $1.0-\mathrm{msec}$ pulses, $60 \mathrm{pps})$ were then delivered at $120-$ to $160-\mathrm{sec}$ intervals. The initial stimulation intensity was 10 microA and was increased by 10 -microA increments until a Stage 4 or 5 motor seizure was evoked. Duration of the Stage 4 or 5 seizure was measured with a stopwatch.

\section{RESULTS AND DISCUSSION}

All electrodes terminated within the amygdaloid complex in a region bounded by the ventral portion of the central nucleus, the medial portion of the lateral nucleus, and the lateral portion of the medial nucleus. There was no discernible effect of electrode placement.

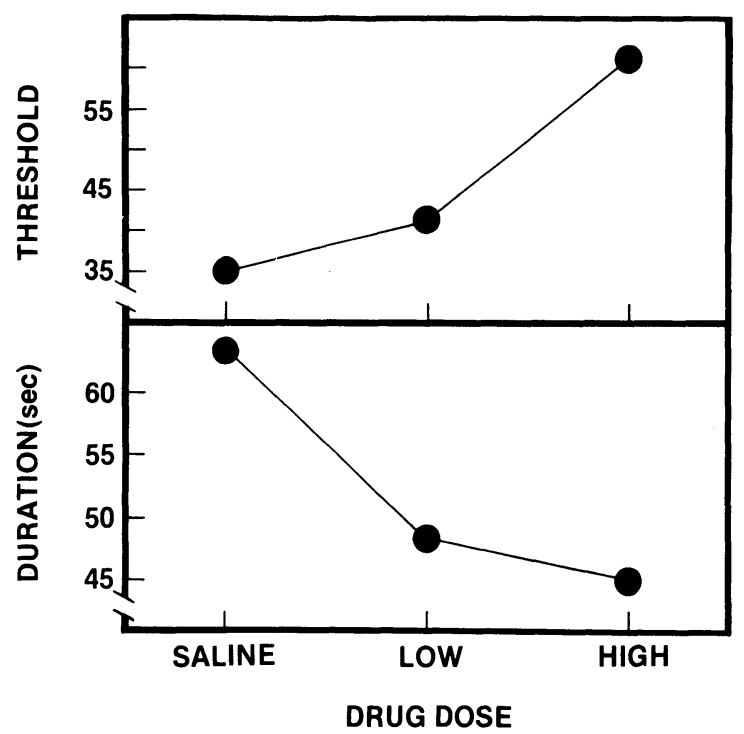

Figure 1. Seizure threshold (upper panel) in microamperes and seizure duration (lower panel) following saline and $.8-\mathrm{g} / \mathrm{kg}$ (low) and $1.6-\mathrm{g} / \mathrm{kg}$ (high) ethanol doses. 
The results of this experiment are summarized in Figure 1. It can be seen (upper panel) that there was a dose-dependent increase in Stage 4 or 5 seizure threshold with increasing ethanol dosage $[\mathrm{F}(2,24)=15.61$, $\mathrm{p}<.01]$. There was no effect of testing order and no interaction between drug dose and testing order (ps $>.10$ ). Seizure duration (lower panel) showed a marginally reliable decline with increasing ethanol dosage $[F(2,24)=2.84, p<.10]$, with no effect of testing order $(\mathrm{p}>.10)$. There was an interaction between drug dosage and testing order, however $[\mathrm{F}(2,24)=3.63$, $p<.05]$. This interaction resulted from a failure of ethanol to depress seizure duration during the first threshold test. Since stimulation was delivered at relatively brief intervals, it might be suspected that the observed ethanol effects resulted from altered responsiveness to lower levels of stimulation. If ethanol increased the frequency of less severe (i.e., Stages 1-3) seizures, this might have inhibited the expression of Stage 4 or 5 seizures. There was no discernible drug effect on the number of Stage 1-3 seizures, however (ps $>.10)$. Further, there was a significant increase in threshold with high (ps $<.005)$ but not with low (ps $>.10$ ) ethanol doses for each of these lower seizure stages. The results of this experiment are thus quite consistent with those of Mucha and Pinel (1979) in demonstrating a short-term attenuation of seizure susceptibility by ethanol.

\section{REFERENCES}

Mucha, R. F., \& Pinel, P. J. Increased susceptibility to kindled seizures in rats following a single injection of alcohol. Journal of Studies on Alcohol, 1979, 40, 258-271.

Pinel, J. P., Van Oot, P. H., \& Mucha, R. F. Intensification of alcohol withdrawal syndrome by repeated brain stimulation. Nature, 1975, 254, 510-511.

RACINE, R. J. Modification of seizure activity by electrical stimulation. II. Motor seizure. Electroencephalography and Clinical Neurophysiology, 1972, 32, 281-294.

(Received for publication August 8, 1980.) 